\title{
INTOXICACIONES AGUDAS EN EL HOSPITAL UNIVERSITARIO DE NEIVA, COLOMBIA, ENTRE EL 2005 Y EL 2010
}

\author{
Acute poisoning at University Hospital in Neiva, Colombia
} from 2005 to 2010

Resumen. Con el objetivo de tener un mejor conocimiento sobre el comportamiento epidemiológico de las intoxicaciones en nuestro medio se realizó un estudio descriptivo, tipo serie de casos con los registros de la base de datos del software SIVIGILA de pacientes con diagnóstico de intoxicación aguda entre el 2005 y el 2010 en el Hospital Universitario. Las variables incluidas fueron: género, edad, procedencia, causalidad de la intoxicación, vía de exposición y tipo de agente causal. Los datos fueron registrados y procesados en el programa Epi Info 2008, versión 3.5.1. Los principales resultados fueron: se encontraron 1347 registros de pacientes con intoxicación aguda. La edad promedio fue $23,4 \pm 11,6$. La principal causa de intoxicación fue la voluntaria $76 \%(\mathrm{n}=1027)$, seguida de la accidental $18 \%$ $(n=236)$. Los casos en la zona urbana fueron el 65\% (n $=875)$ y en la zona rural el 35\% $(n=472)$. Los agentes causales más utilizados fueron los plaguicidas, predominando el grupo de los inhibidores de la colinesterasa, seguido de los medicamentos. El grupo etario más frecuente fue el de 16 a 30 años y no hubo diferencia significativa en ambos géneros. En la procedencia, los municipios con mayor número de casos fueron Neiva, Pitalito y Palermo. Se concluye que hay tendencia al aumento de intoxicaciones en los últimos cinco años, con predominio de las intoxicaciones de tipo voluntario en adolescentes y adultos jóvenes, provenientes en mayor número de la zona urbana y como agente causal se utilizan principalmente los plaguicidas.

Palabras clave: intoxicaciones, epidemiología, plaguicidas, organofosforados, carbamatos.

Abstract. In order to have a better understanding of the epidemiological pattern of poisoning in our area, a descriptive study, case series was carried out. For this purpose it was used the records from the database of Software SIVIGILA of patients classified as confirmed poisoning case from 2005 to 2010 in Hospital Universitario in Neiva. The variables included were: gender, age, origin, causality of poisoning, exposure route and type of causative agent. Data were recorded and processed with Epi Info 2008, version 3.5.1. The main results were: there were 1347 records of patients with acute poisoning. The average age was $23.4 \pm 11.6$. The main cause of poisoning was voluntary $76 \%(n=1027)$ followed by accidental $18 \%(n=236)$. The cases in urban areas were $65 \%(n=875)$ and in rural areas

\footnotetext{
* Médica Toxicóloga clínica. Docente Universidad Surcolombiana, Hospital Universitario de Neiva. Correo electrónico: adrianazamora73@hotmail.com

* Médico Epidemiólogo, Universidad Surcolombiana, Hospital Universitario de Neiva. Correo electrónico: dagosanto@gmail.com
} 
$35 \%(n=472)$. The most common causative agents were pesticides, being the dominated group that of cholinesterase inhibitors, followed by drugs. The most frequent age group was 16 to 30 and no significant difference in both genders was found. In terms of origin, the municipalities with the highest number of cases were Neiva, Pitalito and Palermo. It is concluded that there is an increasing trend of poisoning in the last 5 years, predominantly in voluntary intoxication in adolescents and young adults, coming mainly from urban areas and being pesticides the most used causative agent.

Key words: intoxications, epidemiology, pesticides, organophosphates, carbamates.

\section{INTRODUCCIÓN}

Uno de los objetivos de la toxicología clínica es mantener una información actualizada sobre el perfil epidemiológico de las intoxicaciones agudas. Este conocimiento es imprescindible para favorecer el desarrollo de programas de prevención, diagnóstico y tratamiento. Por otra parte, en los últimos decenios se ha incrementado la disponibilidad y el uso de sustancias químicas potencialmente tóxicas en la industria, el sector agrícola, farmacéutico y en nuestros hogares, con el agravante de la alta frecuencia de manipulación inadecuada, la falta de elementos de protección, el descuido al dejar sustancias tóxicas al alcance de los niños, los hechos suicidas y delictivos, entre otras circunstancias, que provocan cuadros clínicos de origen tóxico que ingresan a diario a los servicios de urgencias de las entidades hospitalarias, convirtiéndose estos lugares en la principal fuente para obtener los datos epidemiológicos ${ }^{(1,2)}$.

Las bases epidemiológicas nacionales como las del Centro de Información y Asesoría Toxicológica (programa que funciona como un convenio entre el Ministerio de la Protección Social, la Universidad Nacional de Colombia y el Consejo Colombiano de Seguridad) han encontrado que los principales agentes causales de intoxicaciones son en su orden: plaguicidas, medicamentos, sustancias cáusticas y corrosivas, derivados de hidrocarburos, bebidas alcohólicas, animales y plantas venenosas ${ }^{(3)}$. Así mismo el Centro de Información de Seguridad sobre Productos Químicos de Colombia, CISPROQUIM, reportó que durante el año 2009 las llamadas para asesoría sobre emergencias toxicológicas fueron 8027 donde más de la mitad de los intoxicados $(55,4 \%)$ eran personas en edad productiva, el $47,3 \%$ de las emergencias eran por intoxicación volunta- ria, seguido por las intoxicaciones accidentales, $29,6 \%$, debido a la exposición no esperada o el uso inadecuado de los productos ${ }^{(4)}$.

El Hospital Universitario Hernando Moncaleano Perdomo (HUHMP) de Neiva es una entidad de tercer nivel que atiende a la población del Huila, el sur del Tolima, el Caquetá, parte del Amazonas, el Putumayo y el sur del Cauca. Motivo por el cual es considerado una fuente importante de datos epidemiológicos sobre la incidencia de las intoxicaciones en el suroccidente del país, para poder posteriormente establecer cuáles son las poblaciones más vulnerables y los pasos a seguir respecto a programas de prevención, control y capacitación tanto para el personal médico y paramédico de la región, como para la comunidad.

\section{MATERIALES Y MÉTODOS}

Para el presente trabajo se realizó un diseño de estudio descriptivo, tipo serie de casos. La población que se tomó fueron los registros de la base de datos del software SIVIGILA del Hospital Universitario Hernando Moncaleano Perdomo (HUHMP), la cual está disponible en la institución desde el año 2005. Por lo cual, el periodo de este estudio se tomó entre el 2005 al 2010. Se definió como caso aquellos registros de la base de datos que fueron clasificados como "caso confirmado" de intoxicación de acuerdo con los protocolos de vigilancia del Instituto Nacional de Salud para eventos de salud pública.

Los casos definidos para el análisis de este estudio fueron: plaguicidas cod 360, fármacos cod 370, metanol cod 380, metales pesados cod 390, solventes cod 400, otras sustancias químicas cod 410 , sustancias psicoactivas cod 414 , accidente ofídico cod 100 y enfermedad transmitida por alimentos cod 355. Se excluyeron aquellos registros en que se descartó o no fue claro el caso de intoxicación o con información incompleta. Se tomaron en cuenta las variables demográficas: género, edad, zona rural o urbana; variables de causalidad: accidental, delictiva, iatrogénica, laboral e intento de suicidio; y vía de exposición: dérmica, inhalatoria, oral y parenteral. Los datos fueron registrados y procesados en el programa Epi Info 2008, versión 3.5.1.

Para el análisis se realizaron estadísticas descriptivas de frecuencias y prevalencias; para variables cuantitativas discretas como edad se tomaron medidas de tendencia central, de dispersión y se estratificó por grupos etarios. Para variables cua- 
litativas como género, causa de la intoxicación, agente causal y zona se realizó análisis bivariado, se usaron pruebas de regresión logística, y para valorar significancia estadística se usó estadístico Chi 2 y p valor $(\alpha-1)$.

Para el análisis de este estudio no se tomó en cuenta la muerte por intoxicación ya que en el software existe un subregistro por falta de notificación de este evento, lo cual afectaría los datos por sesgo en la información por parte del personal de salud.

\section{RESULTADOS}

Se identificó un total de 1347 registros de pacientes con intoxicación aguda en el Hospital Universitario Hernando Moncaleano Perdomo.

Durante el periodo comprendido por el estudio se observó un aumento progresivo de los registros de intoxicaciones; comenzando en el año 2005 con 190 reportes, hasta el año 2009 con 379 casos. Fue más significativo este incremento en los últimos tres años $(\mathrm{p}<0.05)$ (Tabla 1).

La edad promedio de los pacientes fue de $23,4 \pm$ 11,6 . De acuerdo al género se observa que el 51\% $(n=691)$ de los casos corresponden a mujeres y el $49 \%(n=621)$ a hombres. En cuanto a la edad por género no hubo una diferencia significativa (mujeres $21,7 \pm 10$ y hombres 25,2 $\pm 12,6$ ) (Tabla 2).

De la distribución de acuerdo a la zona el 35\% $(\mathrm{n}=472)$ correspondió al área rural y el 65\% $(\mathrm{n}=875)$ al área urbana (Tabla 2).

De acuerdo con la distribución por grupo etario se muestra un mayor número de casos en el
Tabla 1. Frecuencia de intoxicaciones por año en el Hospital Universitario de Neiva (2005-2010).

\begin{tabular}{|lcc|}
\hline Año & Frecuencia & Porcentaje \\
\hline 2005 & 190 & 14,10 \\
2007 & 208 & 15,40 \\
2008 & 259 & 19,20 \\
2009 & 379 & 28,10 \\
2010 & 113 & 8,40 \\
\hline \multirow{2}{*}{ Total } & 1347 & 100,00 \\
\hline
\end{tabular}

grupo comprendido entre 16 y 30 años, seguido por los grupos comprendidos entre los 6 y 15 años y entre los 31 y 45 años. Esta distribución fue similar tanto para el género masculino como para el femenino. Sin embargo, en el análisis de regresión logística de los grupos etarios se observa que la probabilidad de intoxicación a temprana edad en el género femenino fue mayor con respecto al género masculino. OR 2,37 $(1,77-3,19)$ $\mathrm{p}<0,05$ (Figura 1).

Se encontró que los primeros agentes causales de intoxicación fueron los plaguicidas con el 58\% (n $=784$ ), seguidos de los medicamentos con el $12 \%$ ( $n=167)$ y accidente ofídico con el 5\% $(n=74)$.

En los plaguicidas la vía de exposición más frecuente fue la oral con el $95 \%(n=743)$, seguida de la vía inhalatoria $4 \%(n=29)$. En los medicamentos la vía de exposición más frecuente fue la oral con 99\% ( $\mathrm{n}=165)$ (Tabla 3).

La principal causa de intoxicación en los pacientes que ingresaron al servicio de urgencias

Tabla 2. Correlación de casos de acuerdo al género y procedencia en el Hospital Universitario de Neiva (2005-2010).

\begin{tabular}{|lccc|}
\hline & Femenino (\%) & Masculino (\%) & Total \\
\hline Casos & $691(51,3)$ & $656(48,7)$ & $1347(100)$ \\
Edad (DS) & $21,7 \pm 10$ & $25,2 \pm 12,6$ & $23,4 \pm 11,6$ \\
& & & \\
Zona & & & $472(35)$ \\
Rural & $216(45,8)$ & $256(54,2)$ & $875(65)$ \\
Urbana & $476(54,4)$ & $399(45,6)$ & \\
\hline
\end{tabular}






Figura 1. Pacientes intoxicados en el Hospital Universitario de Neiva clasificados por género y grupos etarios (2005-2010).

Tabla 3. Correlación de casos de acuerdo a la vía de exposición y agente causal en el Hospital Universitario de Neiva (2005-2010).

\begin{tabular}{|c|c|c|c|c|c|c|c|c|c|c|}
\hline & \multicolumn{2}{|c|}{ Oral } & \multicolumn{2}{|c|}{ Dérmica } & \multicolumn{2}{|c|}{ Inhalatoria } & \multicolumn{2}{|c|}{ Parenteral } & \multicolumn{2}{|c|}{ Total } \\
\hline & $\mathbf{N}$ & $\%$ & $\mathbf{N}$ & $\%$ & $\mathbf{N}$ & $\%$ & $\mathbf{N}$ & $\%$ & $\mathbf{N}$ & $\%$ \\
\hline Plaguicidas & 743 & 95 & 10 & 1 & 29 & 4 & 2 & 0,3 & 784 & 58 \\
\hline Medicamentos & 165 & 99 & 0 & 0 & 0 & 0 & 2 & 1 & 167 & 12 \\
\hline Solventes & 18 & 75 & 2 & 8,3 & 4 & 17 & 0 & 0 & 24 & 2 \\
\hline Metanol & 2 & 67 & 1 & 33 & 0 & 0 & 0 & 0 & 3 & 0 \\
\hline Sustancias psicoactivas & 3 & 60 & 0 & 0 & 2 & 40 & 0 & 0 & 5 & 0 \\
\hline Transmitida por alimentos & 8 & 100 & 0 & 0 & 0 & 0 & 0 & 0 & 8 & 1 \\
\hline Accidente ofídico & 0 & 0 & 74 & 100 & 0 & 0 & 0 & 0 & 74 & 5 \\
\hline Otras sustancias químicas & 271 & 96 & 10 & 4 & 0 & 0 & 0 & 0 & 281 & 21 \\
\hline
\end{tabular}

fue voluntaria en el $76 \%(\mathrm{n}=1027)$ de los casos, (zona urbana $87 \%(\mathrm{n}=762)$ y zona rural $56 \%(\mathrm{n}=265)$ ) seguida de accidental en $18 \%$ $(n=236)$. En el análisis de regresión logística se observa que la probabilidad de presentar intoxicaciones voluntarias fue cinco veces mayor en el área urbana OR: 5,27 (IC: 4-7) p: 0,00000001. Sin embargo, la causa tipo accidental fue más prevalente en la población rural, 34\% $(n=159)$ de los casos. La probabilidad de haberse presentado intoxicación por causa accidental fue cinco veces mayor en el área rural OR: 5,3 (IC: 3,86-7,21) p: 0,00000001 (Tabla 4). 
Tabla 4. Correlación entre la causalidad y la procedencia en el Hospital Universitario de Neiva.

\begin{tabular}{|lccccc|}
\hline Causa & Rural & Urbana & Total & OR & P \\
\hline Accidental & $159(34)^{*}$ & $77(9)$ & $236(18)$ & $5,3(3,86-7,21)$ & 0,000000001 \\
Delictiva & $13(2,8)^{*}$ & $13(1,5)$ & $26(2)$ & $1,9(0,81-4,34)$ & 0,1 \\
Iatrogénica & $17(3,6)^{*}$ & $11(1,3)$ & $28(2)$ & $2,93(1,3-6,7)$ & 0,004 \\
Laboral & $18(3,8)^{*}$ & $12(1,4)$ & $30(2)$ & $2,85(1,3-6,3)$ & 0,003 \\
Voluntaria & $265(56)$ & $762(87)^{*}$ & $1027(76)$ & $5,27(4-7)$ & 0,00000001 \\
\hline Total & $472(35)$ & $875(65)$ & $1347(100)$ & \\
\hline
\end{tabular}

* Significancia estadística para este grupo.

Otras de las causas que se presentaron en menor número fueron la laboral con $2 \%(\mathrm{n}=30)$, la delictiva con $2 \%(n=26)$ y la iatrogénica con $2 \%$ $(n=28)$ de los casos; la causa iatrogénica y la laboral tuvieron tres veces mayor riesgo de presentación en la zona rural, p < 0,005 (Tabla 4).

Los agentes causales más utilizados en forma voluntaria fueron los plaguicidas con el $64 \%$ (n $=657$ ), seguidos de los medicamentos con el $12 \%$ $(\mathrm{n}=120)$ (Tabla 5).

Los plaguicidas más implicados en este tipo de intoxicación fueron los carbamatos (en especial aldicarb, propoxur y carbofuran) con 59\% $(n=385)$ y los organofosforados (en especial clorpirifos y triclorphon) $24 \%(n=159)$. (Tabla 6). Los medicamentos más consumidos fueron los antidepresivos $12,5 \%(\mathrm{n}=15)$, analgésicos $12,5(\mathrm{n}=15)$ y las fenotiazinas $10 \%(n=12)$ (Tabla 7$)$.

En las intoxicaciones accidentales nuevamente los plaguicidas fueron el agente causal más frecuente con el 35\% ( $n=82)$, seguido por el accidente ofídico, $31 \%(n=74)$ (Tabla 5$)$.

$\mathrm{Al}$ igual que en las intoxicaciones de tipo voluntario, los plaguicidas más implicados en las

Tabla 5. Correlación entre la causalidad (voluntaria o accidental) y el agente causal en el Hospital Universitario de Neiva (2005-2010).

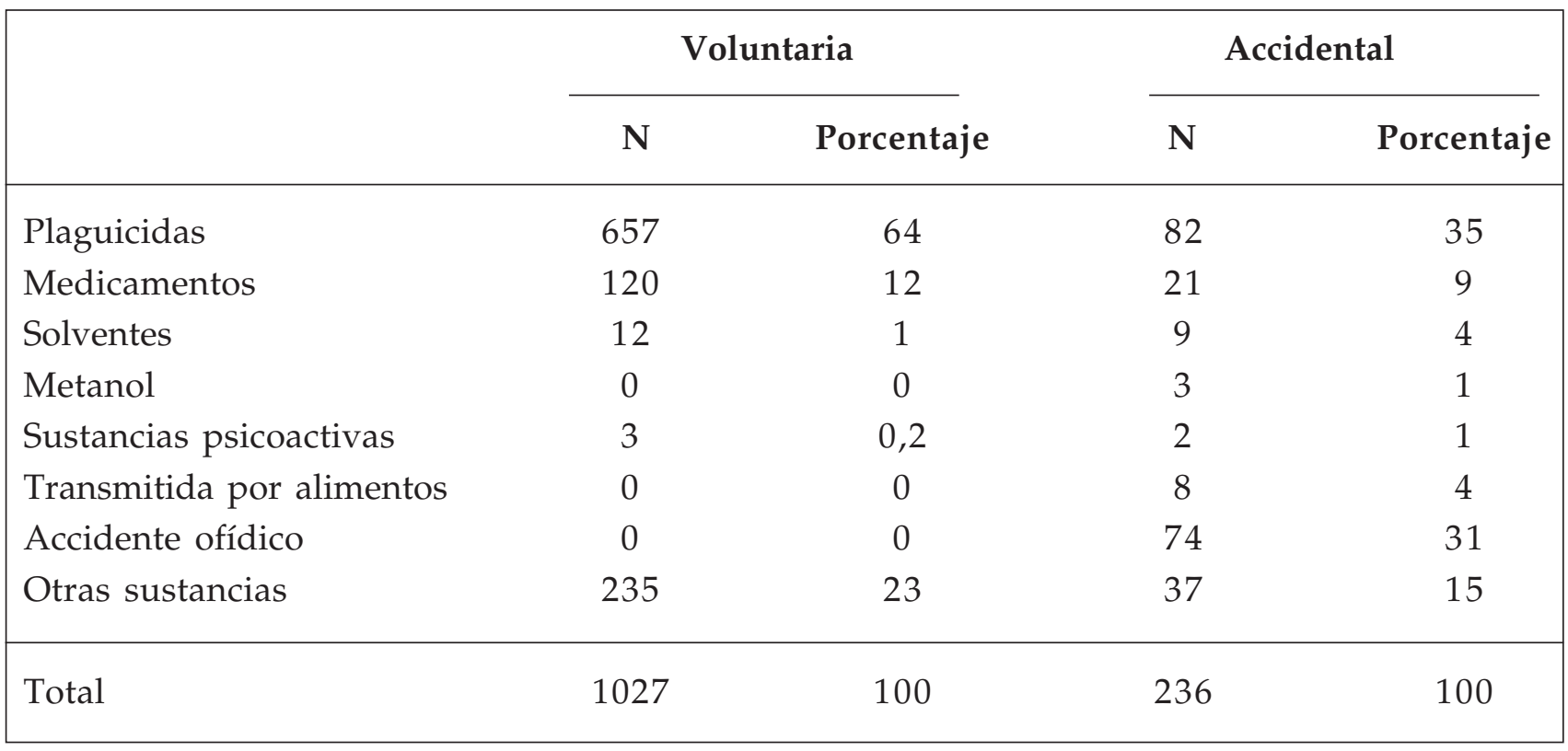


Tabla 6. Correlación entre la causalidad (voluntaria o accidental) y el tipo de plaguicida en el Hospital Universitario de Neiva (2005-2010).

\begin{tabular}{|lcccc|}
\hline & \multicolumn{2}{c}{ Voluntaria } & & \multicolumn{2}{c|}{ Accidental } \\
\cline { 2 - 5 } & N & Porcentaje & N & Porcentaje \\
\cline { 2 - 5 } Carbamatos & 385 & 59 & 34 & 41 \\
Organofosforados & 159 & 24 & 28 & 34 \\
Piretrinas & 41 & 6 & 13 & 16 \\
Amitraz & 30 & 5 & 1 & 1 \\
Organoclorados & 9 & 1 & 0 & 0 \\
Rodenticidas & 5 & 1 & 0 & 7 \\
No especificado & 28 & 4 & 6 & 100 \\
\hline Total & 657 & 100 & 82 & 0 \\
\hline
\end{tabular}

Tabla 7. Correlación entre la causalidad (voluntaria o accidental) y el tipo de medicamento en el Hospital Universitario de Neiva (2005-2010).

\begin{tabular}{|lcccc|}
\hline & \multicolumn{2}{c}{ Voluntaria } & & \multicolumn{2}{c|}{ Accidental } \\
\cline { 2 - 5 } & N & Porcentaje & N & Porcentaje \\
\cline { 2 - 5 } Antidepresivos & 15 & 12,5 & 0 & 0 \\
Analgésicos & 15 & 12,5 & 5 & 23,8 \\
Fenotiazinas & 12 & 10 & 4 & 19 \\
Barbitúricos & 10 & 8,3 & 0 & 0 \\
Benzodiacepinas & 9 & 7,5 & 3 & 14,3 \\
Acetaminofén & 9 & 7,5 & 1 & 5 \\
Anticonvulsivantes & 8 & 6,7 & 0 & 0 \\
Hipotensores & 8 & 6,7 & 2 & 9,5 \\
Sulfato ferroso & 6 & 5 & 3 & 0 \\
Antiparasitarios & 3 & 2,5 & 0 & 0 \\
Varios & 25 & 21 & 0 & 14,3 \\
\hline Total & 120 & 100 & 21 & 00 \\
\hline
\end{tabular}

intoxicaciones accidentales fueron los carbamatos y los organofosforados con el $41 \%$ ( $n=$ 34) y $34 \%(n=28)$ respectivamente (Tabla 6$)$. Los medicamentos más consumidos fueron los analgésicos $24 \%(\mathrm{n}=5)$ y las fenotiazinas $19 \%$ $(\mathrm{n}=4)$ (Tabla 7).
De acuerdo a la distribución por género en las intoxicaciones de tipo accidental encontramos que el 55\% ( $\mathrm{n}=130)$ del total de esta población correspondieron a hombres. En este grupo poblacional el mayor número de casos se encontró en el grupo etario de los 16 a los 30 
años $62 \%(n=81)$. En el género femenino fue similar esta distribución por grupos etarios, encontrándose el mayor número de casos nuevamente en el grupo de los 16 a los 30 años $64 \%(n=68)$, Figura 2. En el análisis de regresión logística se observa que en el grupo etario de 6 a 15 años las mujeres presentaron mayor riesgo de intoxicación respecto a los hombres, $\mathrm{p}<0,05$ OR 2,75 (1,1-7) (Tabla 8).

De acuerdo a la distribución por género en las intoxicaciones de tipo voluntario encontramos que el $52 \%(n=543)$ del total de esta población correspondió a mujeres. En este grupo poblacional

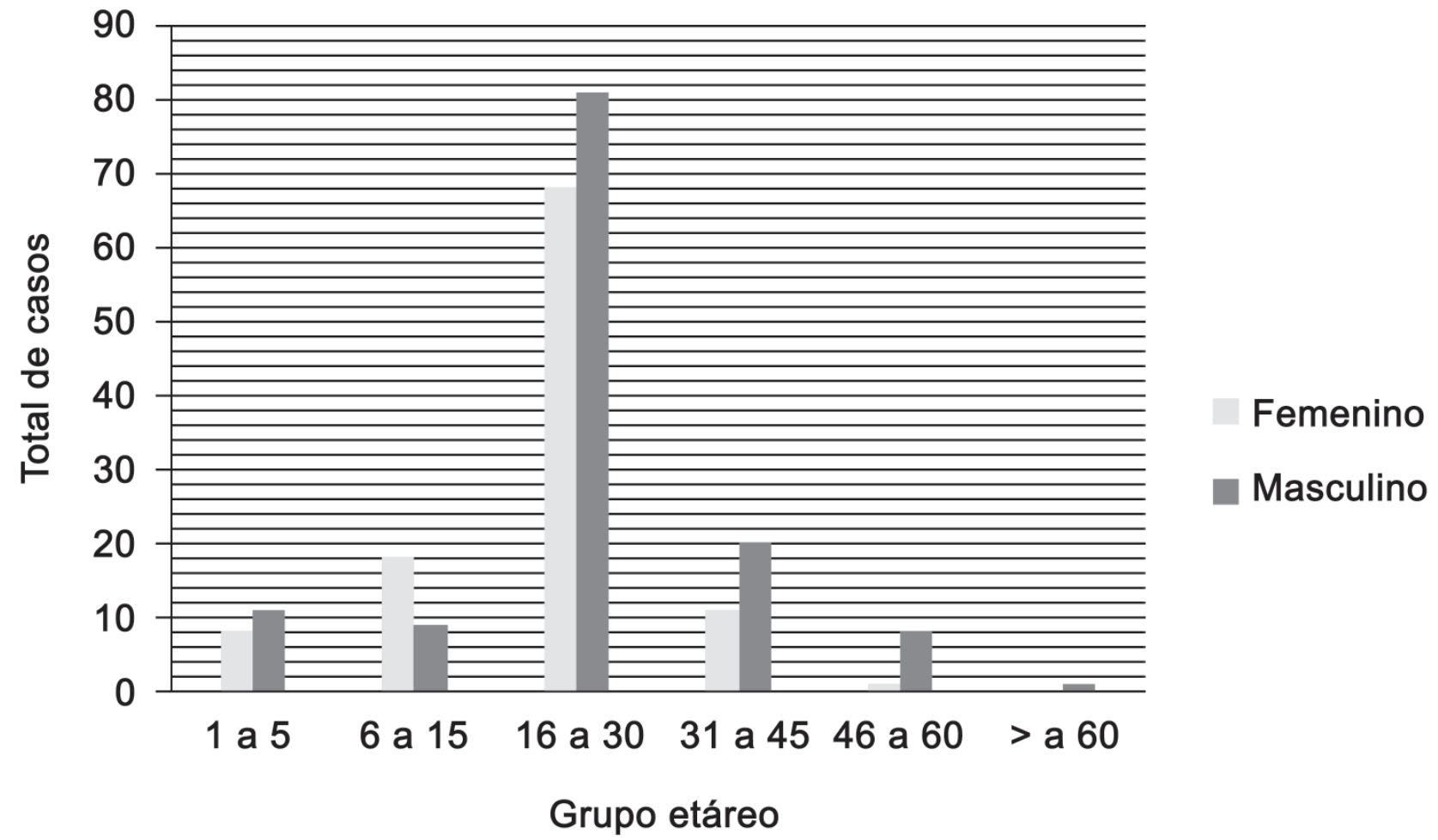

Figura 2. Intoxicaciones accidentales en el Hospital Universitario de Neiva clasificadas por género y grupos etarios (2005-2010).

Tabla 8. Correlación entre género y el grupo etario en las intoxicaciones accidentales en el Hospital Universitario de Neiva (2005-2010).

\begin{tabular}{|lcccc|}
\hline & \multicolumn{3}{c|}{ Accidentales } \\
\cline { 2 - 5 } Grupo etario & Femenino & Masculino & OR & P \\
\hline 1 a5 & $8(8)$ & $11(8)$ & NS & NS \\
6 a 15 & $18^{*}(17)$ & $9(7)$ & $2,75(1,1-7)$ & 0,01 \\
16 a 30 & $68(64)$ & $81(62)$ & NS & NS \\
31 a 45 & $11(10)$ & $20(15)$ & NS & NS \\
46 a 60 & $1(1)$ & $8(6)$ & NS \\
$>$ a 60 & 0 & $1(1)$ & NS & NS \\
\hline Total & $106(45)$ & $130(55)$ & & \\
\hline
\end{tabular}

* Significancia estadística para este grupo. 
el mayor número de casos se encontró en el grupo etario de los 16 a los 30 años $62 \%(n=334)$. En el género masculino fue similar esta distribución por grupos etarios encontrándose el mayor número de casos nuevamente en el grupo de los 16 a los 30 años 57\% (n = 276), Figura 3. En el análisis de regresión logística se observa que las muje- res presentaron mayor riesgo de intoxicación voluntaria respecto a los hombres en los grupos etarios entre 6 y 30 años $p<0,005$. Los hombres presentaron mayor riesgo de intoxicación voluntaria respecto a las mujeres en los grupos etarios de 30 a 60 años. El riesgo fue dos veces mayor con respecto a las mujeres $\mathrm{p}<0,05$ (Tabla 9).

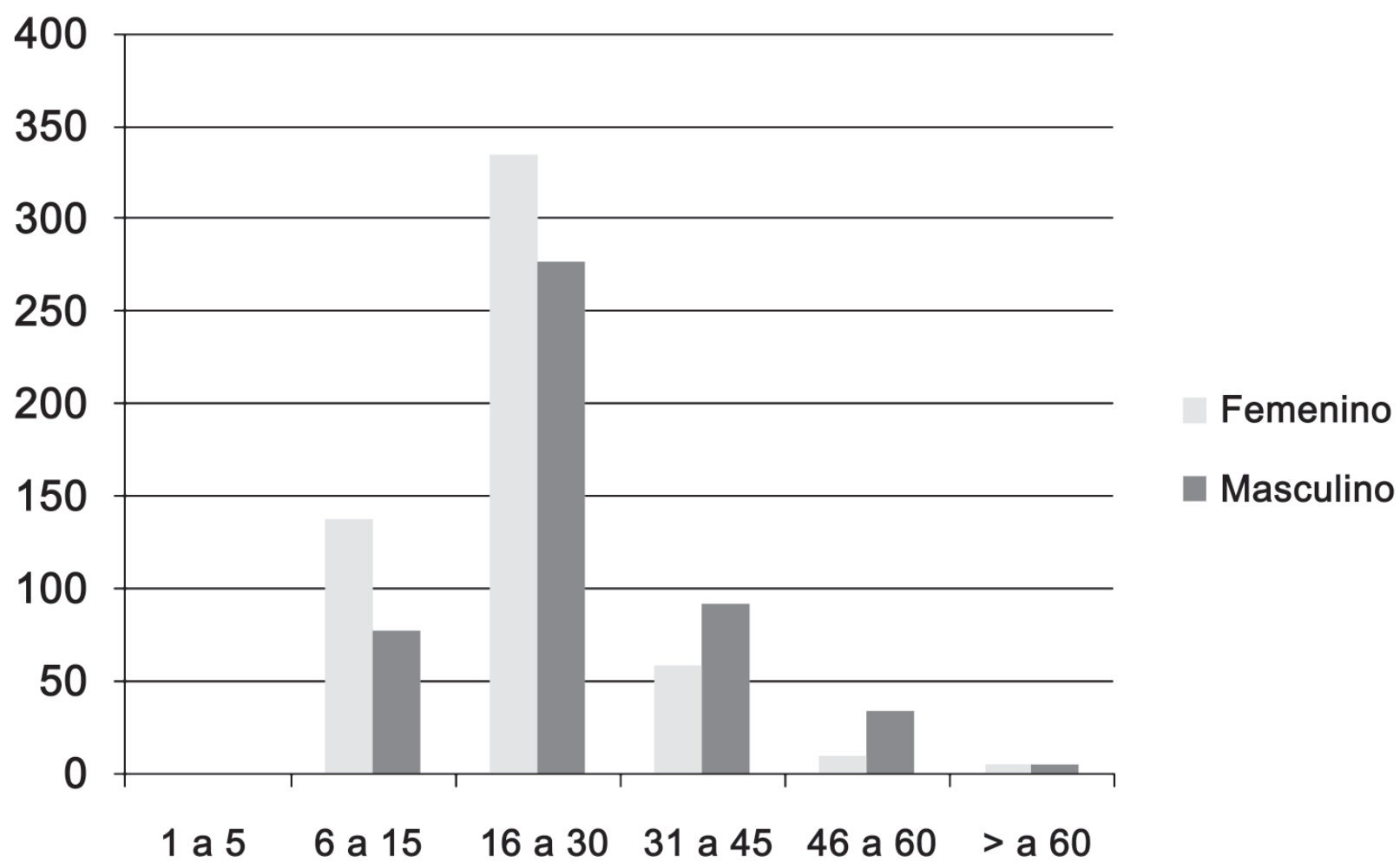

Figura 3. Intoxicaciones voluntarias en el Hospital Universitario de Neiva clasificadas por género y grupos etarios (2005-2010).

Tabla 9. Correlación entre género y el grupo etario en las intoxicaciones voluntarias en el Hospital Universitario de Neiva (2005-2010).

\begin{tabular}{|lcccc|}
\hline & \multicolumn{4}{c}{ Voluntarias } \\
\cline { 2 - 5 } & Femenino & Masculino & OR & P \\
\hline 1 a5 & 0 & 0 & $::$ & $::$ \\
6 a 15 & $137^{*}(25)$ & $77(16)$ & $1,8(1,3-2,5)$ & 0,0002 \\
16 a 30 & $334^{*}(62)$ & $276(57)$ & $1,2(0,9-1,76)$ & 0,14 \\
31 a 45 & $58(11)$ & $92^{*}(19)$ & $2,2(1,52-3,2)$ & 0,00001 \\
46 a 60 & $9(2)$ & $34^{*}(7)$ & $4,5(2-10)$ & 0,00001 \\
$>$ a 60 & $5(1)$ & $5(1)$ & NS & NS \\
\hline Total & $543(52)$ & $484(48)$ & & \\
\hline
\end{tabular}

* Significancia estadística para este grupo. 
En cuanto a la frecuencia de intoxicaciones por municipios se encontró que el mayor número de casos provenían de Neiva $52 \%(\mathrm{n}=696)$, Pitalito $10 \%(n=133)$ y Palermo $5 \%(n=68)$. Los casos provenientes de otros departamentos fueron el $3,3 \%(\mathrm{n}=45)$, que incluyen Tolima, Cundinamarca, Caquetá, Cauca, Nariño y Putumayo (Tabla 10).

Tabla 10. Frecuencia de intoxicaciones por municipio en el Hospital Universitario de Neiva (2005-2010).

\begin{tabular}{|lcc|}
\hline Municipio & Frecuencia & Porcentaje \\
\hline Neiva & 696 & 52 \\
Pitalito & 133 & 10 \\
Palermo & 68 & 5 \\
Campoalegre & 44 & 3,3 \\
Algeciras & 41 & 3 \\
Garzón & 32 & 2,4 \\
Hobo & 31 & 2,3 \\
Aipe & 27 & 2 \\
Acevedo & 26 & 1,9 \\
La Plata & 25 & 1,8 \\
Otros municipios & 179 & 13,3 \\
Otros departamentos & 45 & 3,3 \\
\hline Total & 1.347 & 100,0 \\
\hline
\end{tabular}

\section{DISCUSIÓN}

Este estudio de tipo serie de casos durante cinco años brinda un aporte importante en uno de los eventos de mayor incidencia en el país debido a que existen pocas publicaciones sobre este tópico.

De los resultados podemos observar que la tendencia de los eventos de intoxicaciones agudas en los últimos años ha ido en aumento, con 190 casos en el 2005 a 379 casos en el 2009, y además se observó que los primeros agentes causales de intoxicación fueron los plaguicidas con el 58\% ( $\mathrm{n}=784)$, seguido de los medicamentos con el $12 \%(\mathrm{n}=167)$. Este comportamiento es muy similar con la literatura Nacional ${ }^{(4)}$. Un ejemplo de esto es la incidencia de intoxicación en Colombia en el 2003 que fue de 5,4 por 100.000 hab. y en el 2008 esta cifra aumentó a 43,4 por 100.000 hab. ${ }^{(5)}$. El Huila durante el 2008 y 2009 fue el segundo departamento de mayor inciden- cia en intoxicaciones por plaguicidas ${ }^{(5)}$. Esta incidencia está relacionada con los casos de plaguicidas en nuestro estudio, principalmente en las intoxicaciones voluntarias (64\%) y accidentales (35\%) donde los químicos más implicados fueron los carbamatos (en especial aldicarb, propoxur y carbofuran).

Colombia es un país con un importante renglón en su economía de tipo agrícola, donde los plaguicidas, sin duda, han sido un arma útil y necesaria para el control de las plagas que pueden afectar este renglón del desarrollo. Dentro de este grupo se destacan los inhibidores de la colinesterasa como productos económicos y efectivos, que combaten agentes que otros plaguicidas no han podido controlar ${ }^{(2)}$. Pero, desafortunadamente, la distribución sin control, el fácil acceso y la utilización descuidada han facilitado que se vuelvan los principales agroquímicos implicados tanto en las intoxicaciones con fines suicidas como en las accidentales $(2,6)$.

Los resultados obtenidos muestran como la mayoría de intoxicaciones fueron de tipo voluntario: $76 \%(n=1027)$ de los casos. Sin embargo, existen estudios donde las intoxicaciones de tipo accidental son más frecuentes que las voluntarias y sus agentes causales son diferentes a los plaguicidas. Salas y col. describieron la intoxicación accidental como la más frecuente (62\%) $y$ el principal agente fueron alimentos en inadecuada conservación ${ }^{(7)}$. Ferrer y col. reportaron un mayor predominio de intoxicaciones accidentales por sobredosis de sustancias psicoactivas y medicamentos en un $52 \%{ }^{(1)}$. Mena y col. reportaron un $78 \%$ de intoxicaciones accidentales por medicamentos ${ }^{(8)}$. Silva y col. reportaron que el $72 \%$ de los casos son accidentales y $28 \%$ corresponden a suicidios, en los cuales los agentes causales más frecuentes son los medicamentos $(21,6 \%)^{(9)}$. González y col. publicaron que la intoxicación voluntaria fue la más frecuente, con un 53\% de los casos, predominando el uso de medicamentos ${ }^{(10)}$.

El comportamiento suicida no es exclusivo de los países en desarrollo, pero, de acuerdo con las referencias previamente citadas, se observa que en los países industrializados su incidencia ha disminuido, cediéndole el primer lugar a las intoxicaciones de tipo accidental ${ }^{(1,8,9)}$. Esto es explicable en algunos estudios por el aumento del consumo de sustancias psicoactivas con fines lúdicos, lo que ayuda a aumentar las tasas de accidentabilidad ${ }^{(1,10)}$ en otros estudios, el uso de medicamentos en forma inadecuada ha au- 
mentado la accidentabilidad, desplazando a los suicidios $^{(8-10)}$. Se puede acceder fácilmente a sustancias como antidepresivos, analgésicos tipo paracetamol, antipsicóticos y anticonvulsivantes, ya que la legislación sólo regula la compra, bajo receta médica, de las benzodiacepinas y los opioides ${ }^{(8-10)}$. Esta situación también se presenta en nuestro medio; por esto los medicamentos ocupan el segundo lugar como agentes causales de las intoxicaciones, no solo con fines suicidas y accidentales sino también en las intoxicaciones de tipo delictivo ${ }^{(11)}$.

En las intoxicaciones voluntarias, en los grupos etarios entre los 16 y 30 años, el género femenino y la zona urbana fueron los que mayor número de casos aportaron en el estudio $(\mathrm{p}<0,05)$. Este resultado es similar al de otros artículos donde se encontraron como factores causales una mayor frecuencia de automedicación en mujeres, y el hecho de incluirse en este grupo a los adolescentes, que por sus características psicológicas resultan vulnerables ya que no siempre saben responder a los conflictos propios de su edad y es más probable que utilicen sustancias psicoactivas $^{(9,10)}$. Ferrer y col. encontraron en el grupo de 15 a 30 años una mayor frecuencia, pero a diferencia de nuestros resultados la incidencia fue mayor en hombres $65 \%{ }^{(1)}$.

La alta procedencia urbana difiere de otros estudios donde, por el contrario, se reportó un mayor número de casos en zonas rurales $(4,6,10,12)$. El comportamiento suicida es un fenómeno multifactorial que incluye causas biológicas, psicológicas y sociales ${ }^{(6)}$, y estos factores pueden ser de mayor relevancia en los centros urbanos; sin embargo, es importante realizar estudios complementarios para buscar factores de riesgo que nos permitan sustentar esta situación en este tipo de población.

En las intoxicaciones accidentales el grupo etario de 16 a 30 años y la zona rural son de mayor riesgo (p: 0,05). Esto es explicable por el tipo de actividades que se desarrollan en el campo, donde es mayor la posibilidad de que el contacto con los plaguicidas y otras sustancias químicas se presente dentro del contexto de las labores agrícolas y sin medios de protección que previenen una intoxicación en el momento de la manipulación del producto. Estas actividades usualmente son desarrolladas por el adulto joven ${ }^{(2,12)}$.

Mena y col. reportaron que la mayor causa de intoxicaciones accidentales correspondió a menores de cinco años con el 50\% de los ca- $\operatorname{sos}^{(8)}$. Esto se atribuyó a la curiosidad innata del individuo a temprana edad y la tendencia de llevar a la boca objetos y sustancias como técnica de exploración, sumado al almacenamiento inadecuado de diversas sustancias potencialmente tóxicas, el re-envasamiento a recipientes que puedan ser atractivos para menores y la carencia de envases de seguridad para medicamentos. Esto difiere de nuestros resultados donde, a pesar de presentarse intoxicaciones en este grupo etario no fue el más relevante ( $\mathrm{n}=19$ casos).

De acuerdo con la vía de exposición, la oral fue la más frecuente $(n=1210)$. En las intoxicaciones por plaguicidas esta vía de exposición se presentó en el $95 \%$ de los casos y en los medicamentos en el 99\%. Este resultado coincide con el de la literatura $^{(4,6-10)}$. En las intoxicaciones voluntarias es la vía de acceso más factible para la persona y en las accidentales influyen aspectos como los ya citados previamente sobre la utilización de envases y formas de almacenamiento inadecuados.

\section{CONCLUSIONES}

Un total de 1347 registros de pacientes con intoxicación aguda fueron identificados en el Hospital Universitario Hernando Moncaleano Perdomo. La tendencia durante el tiempo se observa en aumento en los últimos años.

La principal causa de intoxicación en los pacientes que ingresaron al servicio de urgencias fue voluntaria en el 76\% $(n=1027)$ de los casos, seguida de la accidental en $18 \%(n=236)$; las causas laborales, las delictivas y las iatrogénicas tuvieron menor relevancia. El grupo etario más frecuente en intoxicaciones voluntarias y accidentales fue el de 16 a 30 años y no hubo diferencia significativa entre ambos géneros. Sin embargo, la probabilidad de intoxicación a temprana edad en el género femenino fue mayor con respecto al género masculino, $\mathrm{p}<0,05$. La zona urbana fue la que mayor número de casos aportó en intoxicaciones, principalmente de tipo voluntario. $\mathrm{P}<$ 0,05 . Sin embargo, la causa tipo accidental fue más prevalente en la población rural. $\mathrm{p}<0,05$. Los primeros agentes causales de intoxicación fueron los plaguicidas con el 58\% $(\mathrm{n}=784)$ y los medicamentos con el $12 \%(n=167)$. Los plaguicidas más implicados fueron los carbamatos (aldicarb, propoxur y carbofuran) y los organofosforados (clorpirifos y triclorphon). Los medicamentos más consumidos 
fueron los antidepresivos, los analgésicos y las fenotiazinas.

En cuanto a la procedencia de los casos, los municipios que mayor número de casos de intoxicaciones aportaron fueron Neiva con $52 \%(n=696)$, Pitalito $10 \%(n=133)$ y Palermo $5 \%(n=68)$. También se presentaron casos de otros departamentos como Tolima, Cundinamarca, Caquetá, Cauca, Nariño y Putumayo en una menor frecuencia.

\section{RECOMENDACIONES}

La alta incidencia de intoxicaciones asociadas a motivaciones suicidas requiere un abordaje multidisciplinario que incluya no solo el soporte psicológico en la institución de salud sino también el desarrollo de programas de prevención para el suicidio en los primeros niveles de atención.

Se deben desarrollar políticas de regulación sobre la venta y el uso de pesticidas.

Se requiere la implementación de un centro toxicológico en la región, donde se pueda realizar apoyo a los profesionales de la salud en el tratamiento eficaz de las intoxicaciones, teniendo en cuenta la gran diversidad de los posibles agentes y la necesidad de realizar en algunos casos laboratorios químicos para confirmar la sustancia causal.

\section{REFERENCIAS}

1. Ferrer A. Nogué S, Dueñas A, Civeira E. et al. Perfil epidemiológico actual de las intoxicaciones agudas en urgencias. En Intoxicaciones agudas graves. Ars Médica 2006;1-15.

2. Varona M, Henao G, Lancheros A, Murcia A, et al. Factores de exposición a plaguicidas organofosforados y carbamatos en el departamento de Putumayo 2006. Biomédica 2007;27:400-409.
3. Palacio D. Guías para el manejo de urgencias toxicológicas. Grupo de atención de emergencias y desastres. Ministerio de la protección social 2008;17-18.

4. Consejo Colombiano de Seguridad CISPROQUIM. Informe de Emergencias Anual-2009. Www.cisproquim.org.co

5. Ministerio de la Protección Social. Intoxicaciones en Colombia. Base de datos SIVIGILA 2009. www.minproteccionsocial.gov.co

6. Sarmiento MJ, Caballero A. Pesticidas como método suicida: un reporte de caso con Chlorfenapyr. Revista Colombiana de Psquiatría 2008;37(2):272-279.

7. Salas RD, Andrade P, Buena PV. Epidemiología de las intoxicaciones asistidas en la clínica Madre Bernarda (1992-1997), Clínica Vargas (19941997), Clínica Blas de Lezo (1994-1997) y Hospital Univer sitario de Cartagena (19921997). Acta Toxicologíca Argentina 7(2):38-40.

8. Mena HC, Bettini SM, Cerda JP, Concha SF, Paris ME. Epidemiología de las intoxicaciones en Chile: una década de registros. Revista Médica de Chile 2004;132:493-499.

9. Silva R, Wilkins A, Rodriguez L, Olvera R. Panorama epidemiológico de las intoxicaciones en México. Revista Medicina Interna de México 2005;21(2):123-132.

10. González ML, Conill Tomasa, Pérez R. Incidencia de las intoxicaciones agudas. Revista Cubana de Medicina General Integral 1999;15(1):24-31.

11. Uribe M, Moreno C, Zamora A, Acosta P. Perfil epidemiológico de la intoxicación con burundanga en la clínica Uribe Cualla S.A. de Bogotá, D.C. Acta Neurológica Colombiana 2005;21:197-201.

12. Cárdenas O, Silva E, Morales L, Ortiz J. Estudio Epidemiológico de exposición a plaguicidas organofosforados y carbamatos en siete departamentos colombianos 1998-2001. Revista Biomédica 2005;25:170-180. 


\section{UNIVERSIDAD}

SURCOLOMBIANA

FACULTAD DE SALUD 